\title{
Utilização de Mapas no Campo da Epidemiologia no Brasil: Reflexões sobre Trabalhos Apresentados no IV Congresso Brasileiro de Epidemiologia*
}

Luiza Iñiguez Rojas

Universidad de La Habana

Christovam Barcellos

Fundação Oswaldo Cruz

Paulo Peiter

Fundação Oswaldo Cruz

\begin{abstract}
Resumo
A utilização de mapas em epidemiologia tem crescido de forma marcante nos últimos anos. Esta ferramenta, no entanto, não é nova e pressupõe uma forte base teórica e tecnológica. Como forma de avaliar o uso atual de mapas no Brasil, foram levantados os posters apresentados no IV Congresso Brasileiro de Epidemiologia que continham mapas. Estes mapas foram classificados segundo objetivos de ilustração da área de trabalho, demonstração de indicadores de saúde ou análise de dados espaciais. Um total de 131 trabalhos apresentaram mapas, representando $11 \%$ do total de trabalhos, sendo a maior parte destes utilizados como ilustração. Poucos trabalhos utilizaram mapas como meio de análise de eventos sanitários com expressão espacial, grande parte destes concentrados em algumas áreas temáticas e instituições. O acesso a bases de dados gráficos e não-gráficos, bem como a existência de equipes multidisciplinares, podem estar atuando como fatores limitantes ao uso de mapas na epidemiologia. A vigilância em saúde tem sido a maior beneficiária do uso de mapas, talvez por estes permitirem avaliar hipóteses de riscos que envolvem questões ambientais, socioeconômicas e de dinâmica de doenças.
\end{abstract}

\section{Palavras-Chave}

Geografia Médica; Mapeamento em Saúde; Geoprocessamento.

\section{Summary}

The use of maps in epidemiology has been increasing in the last years. Nonetheless, this tool is not new and presupposes solid theoretical and technological basis. The current use of maps in Brazil was assessed by a survey of all posters presented at the IV Brazilian Conference of Epidemiology. The presented maps were classified according to their purposes, either as: illustration of the work area, demonstration of health indicators, or analysis of spatial data. An amount of 131 posters employed maps, representing $11 \%$ of the posters. Illustration was the major purpose of maps and few posters used maps as an analytic tool of sanitary events with spatial expression. A large part of these were concentrated in specific thematic areas and institutions. The access to graphic and non-graphic data bases, as well as the existence of multidisciplinary groups could be acting as limiting factors to the use of maps in epidemiology. Health surveillance has been benefited by the use of maps, due perhaps, to its capability to evaluate risk hypotheses that involve environmental, socioeconomic and disease dynamics factors.

Key Words

Medical Geography; Mapping in Health; Geoprocessing.

\footnotetext{
* Trabalho financiado pelo CNPq (Bolsa de Pesquisa)

Endereço para correspondência: Departamento de Informações em Saúde - DIS/CICT/FIOCRUZ - Centro de Informação em Ciência e Tecnologia, Fundação Oswaldo Cruz, Av. Brasil, 4365, Manguinhos - Rio de Janeiro/RJ - CEP: 21.045-900

E-mail: xris@fiocruz.br
} 


\section{Introdução}

A observação e a interpretação da distribuição dos fenômenos na face da terra têm sido consideradas as principais tarefas dos geógrafos desde a origem desta disciplina no final do século XIX e, para isso, têm sido desenvolvidos métodos e técnicas de mapeamento e, mais recentemente, de interpretação de imagens. A utilização de mapas por geógrafos é tão comum ao ponto de se considerar como a linguagem de expressão da Geografia por excelência, postulando-se por isso que todo geógrafo deva ser também cartógrafo. A geografia, contudo, não pode ser confundida com a cartografia apesar de tradicionalmente utilizá-la como ferramenta e meio de expressão, pois os mapas não são os únicos meios de analisar a espacialidade de um fenômeno. A cartografia, por sua vez, tem avançado velozmente nas duas últimas décadas tornando-se um campo cada vez mais especializado. Os avanços da informática na década de 80 , com o advento dos computadores pessoais, levaram a uma ampla difusão das técnicas de mapeamento digital e geoprocessamento. Na geografia, este fenômeno teve grandes efeitos, levando alguns autores a afirmar que as novas tecnologias de mapeamento e geoprocessamento têm sido responsáveis pela reedição da geografia quantitativa na geografia, agora com a nova roupagem da "análise espacial", tendo na estatística espacial seus principais fundamentos. ${ }^{1}$

Por volta da década de 1960, a utilização dos mapas difundiu-se também em outros campos do conhecimento, como nas atividades de pesquisa e planejamento, adquirindo inúmeras utilidades, principalmente na área de gestão (ambiental e territorial). Muito provavelmente, o crescimento do interesse pelos mapas associa-se ao desenvolvimento das tecnologias automatizadas para sua elaboração, que possibilitam o manejo de grandes volumes de informação e a associação de dados estatísticos no espaço. O resultado tem sido o desenvolvimento vertiginoso dos Sistemas de Informações Geográficas (SIG) e a produção ampliada de literatura e de eventos sobre estes temas.

O uso do geoprocessamento na área de saúde tem história bastante recente, principalmente no Brasil. As primeiras aplicações de geoprocessamento datam da década de 50, utilizando-se computadores de grande porte para o planejamento urbano e, posteriormente, para a análise ambiental. A mudança na maneira de produzir mapas promoveu uma transformação dos paradigmas das ditas ciências da terra. A cartografia tem transformado de uma simples técnica de representação de dados em uma disciplina de análise e simulação de superfícies. ${ }^{2}$ A digitação sistemática de dados, junto à oferta de programas de fácil manipulação e equipamentos de baixo custo e alta capacidade de memória, possibilitaram o aparecimento de uma segunda onda, surgida no final da década de 1980 e abrangendo o início dos anos 1990, do uso de geoprocessamento, que envolveu a área de saúde e ampliou o número de usuários destes sistemas para o mapeamento digital, organização de dados espaciais e produção de mapas temáticos ${ }^{3}$. A aplicação de técnicas de mapeamento e geoprocessamento para pesquisas e ações de saúde tem sido incentivada ${ }^{4,5,6}$ fazendo crer que esta "onda" está ainda em formação e este instrumento será crescentemente utilizado, senão na análise espacial de questões de saúde, ao menos como forma de representação de dados.

Os Sistemas de Informações Geográficas (SIG) têm se destacado como ferramentas de geoprocessamento, principalmente nas análises que envolvem fatores ambientais e epidemiológicos. ${ }^{7} \mathrm{Os}$ SIG têm sido apontados como instrumentos de integração de dados ambientais com dados de saúde, permitindo uma melhor caracterização e mesmo quantificação da exposição (proximidade a fontes de emissão de poluentes, presença de focos de vetores de doença) e seus possíveis resultados, os agravos à saúde. ${ }^{8,9}$ Muitos destes sistemas carecem de meios para análise espacial, definida como a capacidade de manipular dados espaciais de diferentes formatos e obter informações adicionais 
a partir destes. ${ }^{10} \mathrm{~A}$ acoplagem de SIG a ferramentas de análise espacial tem sido reclamada por pesquisadores da área de geoprocessamento. $\mathrm{Na}$ área de saúde, observa-se uma crescente instrumentalização de serviços de saúde em sistemas de geoprocessamento, não acompanhada pela capacitação dos profissionais, tanto para a análise destes mapas como para sua correta redação cartográfica.

A ampliação do uso do geoprocessamento e a reintrodução dos mapas na área de saúde não têm sido acompanhadas da incorporação de conceitos e metodologias geográficas. As análises de dados espaciais não podem ser limitadas ao uso de técnicas estatísticas. Esta análise depende fundamentalmente de um arcabouço teórico necessário para o entendimento da estrutura e processos que caracterizam a construção e dinâmica do espaço e permitem interpretar a distribuição de eventos de saúde. O processo, de incorporação dos mapas na área de saúde não tem tido correspondência no desenvolvimento da 'geografia médica' ou 'geografia da saúde' nos países latino-americanos. ${ }^{11}$ Tais considerações justificam o interesse dos geógrafos da saúde em acompanhar este processo, tentando contribuir da forma mais efetiva possível para a sua consolidação e especialmente apoiar a efetiva utilização dos mapas e da análise espacial de agravos à saúde.

É importante que o uso dos mapas seja acompanhado de um conhecimento cartográfico e estatístico mínimo para reduzir ou eliminar os eventuais erros e imprecisões observados na atualidade, além da ampliação de suas potencialidades. Atualmente, observa-se o crescente interesse por mapas. Contudo, é o manejo dos softwares que recebe a maior atenção enquanto que as implicações teóricas e metodológicas da espacialidade dos problemas de saúde despertam menor interesse. Desta forma, corre-se o risco de que a produção de mapas, mesmo aqueles elaborados para os serviços de saúde, não seja aproveitada para a realização das análises das problemáticas de saúde, e como já tem sido colocado: "O processo técnico se adiante à criação e à teoria".
O objetivo deste trabalho é verificar como vêm sendo utilizados mapas nos estudos epidemiológicos no Brasil, usando como exemplo os 1.174 posters apresentados por ocasião do IV Congresso Brasileiro de Epidemiologia, realizado no Rio de Janeiro em julho de 1998. Este tipo de levantamento não é disponível para congressos anteriores nacionais de epidemiologia e outros no exterior, impedindo a análise da evolução histórica e setorial de uso de mapas. Além disso, não foi avaliado o conteúdo dos trabalhos apresentados e a adequação de metodologias de análise espacial para os estudos epidemiológicos. O objetivo principal do levantamento foi análise do grau de complexidade destes mapas, sua construção e inserção nos estudos epidemiológicos.

\section{Metodologia}

Durante a seção de posters no IV Congresso Brasileiro de Epidemiologia, foram levantados os trabalhos que apresentavam qualquer tipo de mapa. Estes mapas foram classificados segundo três categorias de complexidade:

1) O m apa com o ilustração da área de trabalho. Nessa categoria foram incluídas figuras com a localização do estado ou município em que se desenvolveu o trabalho, ou locais de amostragem. O mapa teve como principal função a localização de elementos espaciais de algum significado no trabalho.

2) O mapa como demonstração de indicadores desenvolvidos no trabalho. Nesta categoria está incluída a maior parte dos mapas temáticos encontrados durante a seção. O mapa, nesse caso, teve como função a visualização de resultados gerados pelo trabalho, não sendo comentado ou não contribuindo para a explicação de eventos de saúde. Neste caso, o mapa mais que ilustração, serve de apoio na apresentação de resultados.

3) O mapa como meio de análise de dados espaciais. Foram considerados nessa categoria os mapas que permitiram a obtenção de novas informações que contribuíram para o entendimento do problema de saúde estudado. Incluíramse aqueles com análises realizadas por 
associação visual (de superposição) ou através de tratamento estatístico de dados espaciais. O espaço é nestes casos objetivo central ou um dos objetivos do trabalho, considerando-se como meio para o conhecimento e análises de eventos de saúde.

Os trabalhos foram grupados a partir da divisão em temas propostos pela organização do congresso: avaliação; ensino; espaço e tempo; métodos e técnicas em epidemiologia; morbidade; mortalidade; nutricional; saúde ambiental; saúde bucal; saúde da mulher; saúde infantil; saúde mental; saúde ocupacional; sistemas de informação; terceira idade; vigilância de doenças não transmissíveis; vigilância de doenças transmissíveis; vigilância epidemiológica; e "outros". ${ }^{12}$ Dentro da categoria "outros" encontravam-se trabalhos que foram enviados com atraso para a organização do congresso. Estes trabalhos foram reclassificados dentro dos temas principais do congresso.

\section{Resultados e discussão}

Foram contabilizados 131 trabalhos que utilizaram mapas na seção de poster do congresso, o que representa $11 \%$ do total de trabalhos apresentados. A Tabela 1 mostra a distribuição geográfica, por estados brasileiros, da origem destes trabalhos.

Observa-se, segundo a tabela, a difusão do uso de mapas em grande parte dos estados brasileiros, com uma maior participação de estados que contam com núcleos de pesquisa dotados de núcleos de geoprocessamento e que investiram durante esta década na formação de profissionais e sistemas capazes de manipular dados espaciais. Pernambuco, por exemplo, apresentou 26 trabalhos, sendo nove de análise espacial. Neste estado destaca-se a presença do Centro de Pesquisas Aggeu Magalhães da Fundação Oswaldo Cruz (CPqAM/ FIOCRUZ), da Secretaria Estadual de Saúde e de Secretarias Municipais de Saúde, principalmente de Olinda, com contribuição importante no total dos trabalhos apresentados. No Rio de Janeiro, também a FIOCRUZ foi responsável pela maior parte dos trabalhos que utilizaram os mapas como ferramenta de análise. Minas Gerais, que apresentou uma alta frequiência de mapas nos trabalhos apresentados, utilizou-os principalmente com caráter ilustrativo.

Em 67 trabalhos (51\%), os mapas foram utilizados como ilustração (categoria 1), 37 (28\%) como representação de dados (categoria 2), e

Tabela 1 - Uso de mapas nos posters apresentados durante o V Congresso Brasileiro de Epidemiologia segundo o Estado de origem do trabalho e grau de complexidade

\begin{tabular}{|c|c|c|c|c|c|}
\hline Estado & Ilustração & Demonstração & Análise & Total & Percentagem \\
\hline PE & 10 & 7 & 9 & 26 & 19,8 \\
\hline RJ & 13 & 1 & 6 & 20 & 15,3 \\
\hline MG & 12 & 1 & 2 & 15 & 11,5 \\
\hline SP & 8 & 5 & 2 & 15 & 11,5 \\
\hline BA & 3 & 5 & 1 & 9 & 6,9 \\
\hline $\mathrm{CE}$ & 3 & 6 & 0 & 9 & 6,9 \\
\hline $\mathrm{SC}$ & 5 & 1 & 0 & 6 & 4,6 \\
\hline RS & 1 & 3 & 1 & 5 & 3,8 \\
\hline AM & 1 & 0 & 2 & 3 & 2,3 \\
\hline MT & 1 & 1 & 1 & 3 & 2,3 \\
\hline RO & 0 & 3 & 0 & 3 & 2,3 \\
\hline PA & 1 & 1 & 0 & 2 & 1,5 \\
\hline PR & 1 & 1 & 0 & 2 & 1,5 \\
\hline $\mathrm{AL}$ & 1 & 0 & 0 & 1 & 0,8 \\
\hline MS & 1 & 0 & 0 & 1 & 0,8 \\
\hline TO & 0 & 0 & 1 & 1 & 0,8 \\
\hline $\mathrm{BR}^{*}$ & 5 & 2 & 2 & 9 & 6,9 \\
\hline Total** & 67 & 37 & 27 & 131 & 100,0 \\
\hline
\end{tabular}

* Algumas instituições apresentaram trabalhos tendo como área de abrangência o território nacional.

** Foi apresentado um trabalho desenvolvido na Argentina, e o uso de mapas foi classificado como ilustrativo.

Fonte: Livro de Resumos e Informações obtidas diretamente dos posters (Abrasco, 1998) 
Luiza Iñiguez Rojas e cols.

Tabela 2 - Uso de mapas nos posters apresentados durante o V Congresso Brasileiro de Epidemiologia segundo tema do trabalho e grau de complexidade

\begin{tabular}{|c|c|c|c|c|c|c|}
\hline Tema & [lustração & Demonstração & Análise & Total & $\begin{array}{c}\text { Total de Trabalhos } \\
\text { Apresentados } \\
\end{array}$ & $\begin{array}{l}\text { Percentual de Trabalhos } \\
\text { que apresentaram mapas }\end{array}$ \\
\hline Vigilância transmissível & 15 & 11 & 8 & 34 & 230 & 14,8 \\
\hline Vigilância epidemiológica & ca 14 & 5 & 1 & 20 & 90 & 22,2 \\
\hline Mortalidade & 6 & 6 & 6 & 18 & 82 & 22,0 \\
\hline Sistemas de informação & 2 & 6 & 3 & 11 & 50 & 22,0 \\
\hline Espaço e tempo & 0 & 1 & 8 & 9 & 15 & 60,0 \\
\hline Saúde ocupacional & 5 & 1 & 0 & 6 & 76 & 7,9 \\
\hline Avaliação & 6 & 0 & 0 & 6 & 40 & 15,0 \\
\hline Nutricional & 4 & 0 & 0 & 4 & 78 & 5,1 \\
\hline Saúde ambiental & 3 & 1 & 0 & 4 & 20 & 20,0 \\
\hline Saúde infantil & 3 & 1 & 0 & 4 & 64 & 6,3 \\
\hline Ensino & 2 & 1 & 0 & 3 & 28 & 10,7 \\
\hline Outros & 3 & 0 & 0 & 3 & 222 & 1,4 \\
\hline Saúde bucal & 2 & 1 & 0 & 3 & 38 & 7,9 \\
\hline Morbidade & 0 & 2 & 0 & 2 & 23 & 8,7 \\
\hline Mulher & 1 & 0 & 1 & 2 & 55 & 3,6 \\
\hline Vigilância não transm. & 0 & 1 & 0 & 1 & 41 & 2,4 \\
\hline Saúde mental & 1 & 0 & 0 & 1 & 22 & 4,5 \\
\hline Total & 67 & 37 & 27 & 131 & 1.174 & 11,2 \\
\hline
\end{tabular}

Fonte: Livro de Resumos e informações obtidas diretamente dos posters.

Observação: nenhum mapa foi apresentado nas seções métodos e técnicas, e epidemiologia da terceira idade.

$27(20 \%)$ como meios de análise (categoria 3) de eventos sanitários com expressão espacial (Tabela 2).

A maior parte dos trabalhos (42\%) que utilizaram mapas foram classificados dentro das áreas temáticas de vigilância, sendo a maioria dos mapas de caráter ilustrativo. Na área de vigilância de doenças transmissíveis houve um considerável uso de mapas para a análise de dados epidemiológicos. Por outro lado, a área de vigilância de doenças não transmissíveis apresentou um número restrito de mapas, mesmo se ponderados os totais de trabalhos apresentados em cada área. Grande parte da tradição da geografia médica no mundo está relacionada à identificação de padrões espaciais de distribuição de doenças transmissíveis. ${ }^{13}$ A própria investigação do surto de cólera em Londres por John Snow utilizou mapas para caracterização de fontes de contaminação e marcou o início da epidemiologia com bases científicas. Mais recentemente, no entanto, os epidemiologistas, têm se voltado para a busca de fatores de risco e aglomerados (“clusters") de doenças não-transmissíveis no espaço. ${ }^{14}$ Este é o caso do desenvolvimento de técnicas de análise estatística espacial voltados para a avaliação da existência de casos de câncer em torno de usinas eletronucleares, locais de disposição de lixo tóxico e linhas de transmissão de energia. ${ }^{15,16}$

A área de estudos de mortalidade utilizou com freqüência mapas para ilustração, demonstração e análise de informações. A crescente disponibilidade de dados de mortalidade e a relativa facilidade de georreferenciamento destes dados na escala municipal pode estar sendo um fator de incentivo ao uso de mapas temáticos para a análise epidemiológica espacial.

Grande parte $(60 \%)$ dos trabalhos apresentados sob o tema espaço e tempo em epidemiologia utilizou mapas como ferramentas de análise espacial. Nessa seção se concentram os trabalhos de discussão metodológica sobre espaço e saúde. Destacam-se, nessa área, os trabalhos apresentados por secretarias de saúde em colaboração com instituições de pesquisa. Em outras áreas como a vigilância epidemiológica, saúde ocupacional, ambiental, nutricional e avaliação, os mapas foram utilizados quase exclusivamente para ilustração. É surpreendente a escassez de mapas nas áreas de saúde ambiental e saúde 
ocupacional e quando existiam tinham fins meramente ilustrativos. Este fato contraria a tendência mundial de utilização do geoprocessamento como ferramenta para a organização e análise conjunta de dados ambientais e de saúde. ${ }^{5}$ Um número considerável de trabalhos apresentados em outras áreas temáticas usou mapas para caracterizar áreas de risco e extrair do mapa informações que permitissem um melhor entendimento dos problemas de saúde nestas áreas.

É importante observar que a grande maioria dos mapas apresentados nos trabalhos do congresso eram digitais, isto é, foram construídos em computador, através da importação de figuras que acompanham alguns softwares gráficos, da digitalização de figuras existentes em forma analógica ou da reprodução de mapas obtidos de outras fontes. Este fato aponta para uma crescente apropriação das novas tecnologias de representação cartográfica disponíveis, ainda que em graus distintos.

A disponibilidade de mapas em formato digital só garante a utilização do mapa como "ilustração" (categoria 1 na tabela 2). Este tipo de mapa é 'vazio' de dados, isto é, não está relacionado com nenhuma informação produzida ou trabalhada na área de saúde. Os mapas "demonstrativos" de situações de saúde (categoria 2) dependem da construção de indicadores a partir de dados epidemiológicos, muitas vezes obtidos a partir de bases de dados de saúde e seu georreferenciamento. É necessário um grande esforço para a compatibilização de dados tabulares com bases cartográficas para que estes indicadores sejam apresentados na forma de mapas. ${ }^{17} \mathrm{Um}$ passo inicial neste trabalho é a escolha de uma unidade espacial mínima de agregação de dados e a subseqüente opção por uma escala de trabalho. As dificuldades de georreferenciamento de dados e a disponibilidade de bases cartográficas depende grandemente da unidade espacial de referência escolhida ${ }^{7}$. Paradoxalmente, mapas de grande escala (para o enfoque de problemas locais) tendem a ser mais dificilmente obtidos, enquanto para a análise de problemas de saúde no nível nacional (menor escala), os dados epidemiológicos, demográficos e cartográficos são de mais fácil acesso. Esta disponibilidade de dados não elimina um trabalho árduo de atualização de unidades administrativas, em permanente mudança, nos sistemas de informação. Basta citar a permanente emancipação de municípios (cerca de 140 por ano nessa década) que necessitam ser incorporados

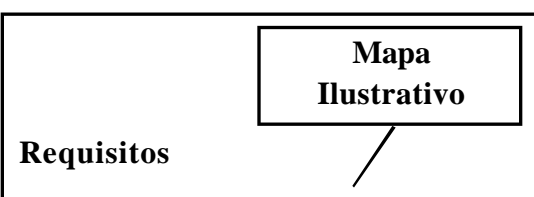

- Aquisição de mapa por processo de reprodução.

- Uso de softwares de edição de figuras.

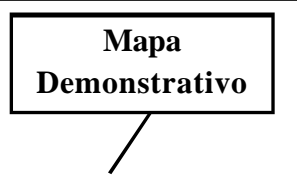

- Disponibilidade de mapa em formato digital.

- Disponibilidade de bases de dados.

- Georreferenciamento de dados.

- Construção de indicadores.

- Uso de softwares simples geoprocessamento.

- Conhecimento de cartografia temática.

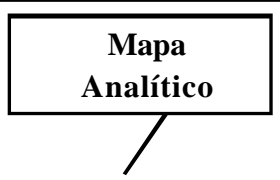

- Formulação de hipóteses espaciais.

- Conhecimento do lugar.

- Conhecimento da etiologia.

- Domínio de técnicas estatísticas e de SIG.

- Uso de softwares para análise especial.

- Conhecimento de cartografia.

Figura 1 - Nível de complexidade de mapas e requisitos teóricos e tecnológicos para sua implementação na área de saúde 
aos sistemas de informação em saúde e à base cartográfica. A escolha desta escala também condiciona os possíveis resultados visuais e estatísticos obtidos na análise de mapas. ${ }^{18,19}$ É uma opção, consciente ou não, do pesquisador ou planejador, a unidade espacial de agregação de dados, que deverá ser compatível com o fenômeno a ser analisado e as ações a serem planejadas. ${ }^{7}$

A análise ( $3^{\mathrm{a}}$ categoria) desses mapas pressupõe uma base teórica para a elaboração de hipóteses envolvendo a relação entre espaço e saúde, um conhecimento do lugar e do problema de saúde, bem como o domínio de instrumentos teórico-metodológicos (onde se incluem a estatística e os SIG). O cumprimento destes requisitos exige, portanto, a formação de equipes multidisciplinares e interinstitucionais. A Figura 1 mostra os diversos pressupostos metodológicos e tecnológicos geralmente exigidos para a construção de mapas com diferentes níveis de complexidade.

\section{Considerações finais}

O geoprocessamento apresenta um enorme potencial nos estudos de saúde mas, esta ferramenta não tem sido utilizada em sua plenitude pela epidemiologia no Brasil como demonstrou a pesquisa em foco. A maior parte dos trabalhos apresentados não utilizou mapas ou o fez como ilustração. Os trabalhos que utilizaram mapas como ferramenta de análise tiveram origem em grupos de pesquisa, concentrados em algumas poucas instituições e estados brasileiros. Por outro lado, já é visível a crescente incorporação das novas técnicas cartográficas e estatísticas computadorizadas na área da saúde, graças ao acesso facilitado às bases de dados epidemiológicos e geográficos, bem como a difusão de softwares simples de geoprocessamento.

O uso destas ferramentas pressupõe a utilização de modelos de explicação do processo saúde/doença, que envolve questões geográficas, como a organização espacial, social e condições ambientais. Contudo, verifica-se que o espaço é compreendido em inúmeros trabalhos de epidemiologia unicamente como um plano geométrico para a disposição e análise estatística de dados. Em outra linha de trabalho, o espaço tem sido fragmentado para permitir verificar associações entre indicadores epidemiológicos e condições sociais e ambientais, incorporando técnicas do estudo ecológico. Estas diferentes abordagens pressupõem e advêm de bases teóricas e metodológicas muitas vezes ocultas nos estudos epidemiológicos ${ }^{19}$. O uso de agregados espaciais pressupõe a independência entre divisões territoriais, enquanto a utilização de técnicas de estatística espacial apresenta, ao contrário, como entendimento prévio, o relacionamento entre elementos espaciais vizinhos. Cada uma destas abordagens apresenta vantagens operacionais e restrições de uso inerentes à concepção de espaço. As técnicas de interpolação de dados espaciais, por exemplo, criam muitas vezes 'manchas' homogeneizadoras que, ao invés de salientar, mascaram os diferenciais espaciais.

Em termos de capacidade técnica e infra-estrutura, o uso de mapas em epidemiologia exige hoje uma estrutura mínima que pode ser inviável para alguns setores de serviço e grupos de pesquisa. O desenvolvimento de técnicas de geoprocessamento e a incorporação de métodos geográficos de análise espacial demanda um suporte de software, equipamentos e pessoal especializado, bem como o trabalho em equipes multidisciplinares. A disponibilidade de bases de dados epidemiológicos e socioeconômicos atualizados pode ser um fator limitante para o desenvolvimento de trabalhos utilizando mapas no nível local. Poucos estados e cidades do Brasil possuem bases cartográficas digitais disponíveis, exigindo a articulação entre instituições de pesquisa e de governo para a construção integrada de SIG. Uma alternativa à falta de dados espaciais tem sido a construção pelo setor de saúde de bases cartográficas próprias como, por exemplo, em Olinda (CPqAM), Porto Alegre (Secretaria Municipal de Saúde) e Rio de Janeiro (FIOCRUZ), exigindo 
nesse caso um esforço ainda maior para contratação de serviços, aquisição de equipamentos, etc.

Os mapas podem gerar produtos de análise importantes para a gestão de saúde como o diagnóstico de problemas de saúde e a locação otimizada de recursos. No entanto, a plena utilização dos mapas como meio de análise de processos espaciais exige hoje uma série de requisitos teóricos e tecnológicos talvez muito pesados para grande parte das instituições de pesquisa e serviço no Brasil. A popularização dos mapas, verificada nesse trabalho, representa aparentemente uma tendência crescente do uso do espaço e de técnicas de geoprocessamento na área da saúde, que será provavelmente acompanhada pelo incremento na demanda de bases de dados cartográficos, epidemiológicos, sociodemográficos e ambientais. $\mathrm{O}$ uso de mapas, nesse caso, torna-se além de fascinante, custoso, pressupondo a incorporação de novas tecnologias e metodologias, em geral ausentes no setor.

\section{Bibliografia}

1. Castro MC. Geoprocessamento. Geografia Quantitativa. De volta para o futuro? Anais da $1^{\text {a }}$ Semana Estadual de Geoprocessamento. Forum Estadual de Geoprocessamento do Rio de Janeiro; 1996; Rio de Janeiro. p. 19-31.

2. Rimbert S. Géographie et cartographie. In: Bailly A, Ferras R, Pumain D. Encyclopédie de Géographie. Paris: Ed. Economica; 1993. p.89-115.

3. Birkin M, Clarke G, Clarke M, Wilson A. Intelligent GIS, location decisions and strategic planning. New York: John Wiley and Sons; 1996. p.21-54.

4. Scholten HJ, Lepper MJC. The benefits of application of geographical information systems in public and environmental health. World Health Statistical Quarterly 1991; 44:160170.

5. Croner CM, Sperling J, Broome FR. Geographic information systems (GIS): New perspectives in understanding human health and environmental relationships. Statistics in Medicine 1996; 15:1961-1977.

6. Castillo-Salgado C. Uso de sistemas de información geográfica en epidemiologia. Boletin Epidemiológico de la Organizacion Panamericana de Salud 1996; 17(1):1-6.

7. Barcellos C, Santos SM. Colocando dados no mapa: a escolha da unidade de agregação e integração de bases de dados em saúde e ambiente através do geoprocessamento. Informe Epidemiológico do SUS 1997; VI(1):21-29.

8. Briggs DJ. Mapping Environmental Exposure. In: Elliot P, Geographical and Environmental Epidemiology: Methods for Small-area Studies. Tóquio: Oxford University Press; 1992. p.158-176.

9. Peiter P, Tobar C. Poluição do ar e condições de vida: uma análise geográfica de riscos à saúde em Volta Redonda, Rio de Janeiro, Brasil. Cadernos de Saúde Pública 1998; 14(3):473-485.

10. Bailey TC. A review of statistical spatial analysis in geographical information systems. In: Fotheringham S \& Rogerson P. Spatial Analysis and GIS. Londres: Ed. Longman; 1994. p. 13-44.

11.Rojas LI. Geografia y salud: temas y perspectivas en America Latina. Cadernos de Saúde Pública 1998; 14(4):701-711.

12.ABRASCO. Livro de resumos. IV Congresso Brasileiro de Epidemiologia. Rio de Janeiro: ABRASCO; 1998.

13.Picheral H, Salem G. De la Géographie Medicale à la Géographie de la Santé. Bilan et Tendances de la Géographie Française (1960 - 1991). Montpellier: Cahiers Geo 22; 1992.

14. Verhasselt Y. Geography of health: some trends and perspectives. Social Science and Medicine 1993; 36(2):119-123.

15.Hills M, Alexander F. Statistical 
methods used in assessing the risk of disease near a source of possible environmental pollution: a review. Journal of Royal Statistical Society Association 1989; 152:353-363.

16.Wartenberg D, Greenberg M, Lathrop R. Identification and characterization of populations living near high-voltage transmission lines: a pilot study. Environmental Health Perspectives 1993; 101:626-632.

17.Guerra H, Carvalho MS.
Geoprocessamento em epidemiologia. Relatório de oficina de trabalho do EPIRIO-98. Rio de Janeiro: ABRASCO; 1998.

18. King PE. Problems of spatial analysis in geographical epidemiology. Social Science \& Medicine 1979; 13:249252.

19. Barcellos C, Bastos FI. Geoprocessamento, ambiente e saúde, uma união possível? Cadernos de Saúde Pública 1996; 12(3):389-397. 